\title{
Progress in Gravitational Wave Detection: Interferometers
}

\author{
Kazuaki Kuroda $^{\mathrm{a}}{ }^{*}$ and LCGT Collaboration ${ }^{\mathrm{b}}$ \\ anstitute for Cosmic Ray Research, The University of Tokyo, \\ 5-1-5, Kashiwanoha, Kashiwa, Chiba 277-8582, Japan \\ b National Astronomical Observatory, High Energy Accelerator Research Organization, \\ Physics Department-The University of Tokyo, \\ Department of Advanced Materials Science-The University of Tokyo, \\ ILS-University of Electro-Communications, ERI-The University of Tokyo, \\ Miyagi University of Education, Metrology Institute of Japan, \\ Department of Earth \& Space Science-Osaka University, YITP-Kyoto University, \\ Physics Department-Niigata University
}

A gravitational wave (GW) is a physical entity of space-time derived from Einstein's theory of general relativity. Challenging projects to observe gravitational waves are being conducted throughout the world. A Japanese project involving a $300 \mathrm{~m}$ baseline laser interferometer, TAMA, achieved $1000 \mathrm{hr}$ of continuous observation with the best sensitivity in the world during the summer of 2001. After achieving promising results, the realization of LCGT (Large-scale Cryogenic Gravitational wave Telescope) will become possible in the near future.

\section{Introduction}

A gravitational wave is a physical entity of spacetime derived from Einstein's theory of general relativity. The existence of a GW was indirectly proved by an observation of PSR 1913+16 by Hulse and Taylor [1]. It is a transverse wave traveling with the speed of light. Sources of GWs are the coalescence of binary neutron stars (BNS), supernova explosions, the coalescence of binary black holes (BBH), stars falling into black holes, spinning neutron stars, orbiting binary stars, cosmological background, vibration of a cosmic string, and so on. Among these sources, the major targets of ground-based laser interferometers are the coalescence of BNS and supernova explosions.

Challenging projects to observe GWs are being conducted throughout the world. Historically, resonant-mass GW antennae have been developed to detect these GW events and a world-wide network of these antennae (IGEC) is watching for GWs [2]. Here, we focus attention on detection

\footnotetext{
*kuroda@icrr.u-tokyo.ac.jp
}

using laser interferometers. When a GW passes through space where two masses are located, the distance between them changes. A suspended mirror $\left(\mathrm{M}_{\mathrm{x}}\right)$ and a suspended beam splitter (BS), which compose a part of a Michelson laser interferometer, behave as test masses for the GW (in Fig. 1). The interferometer measures the differential displacement between two arms in the optical phase. A typical GW occurring in the Virgo cluster could cause a displacement of $10^{-18} \mathrm{~m}$ for a $1 \mathrm{~km}$ baseline length on the Earth. To effectively detect this displacement, optical paths of the laser beam are folded many times. The delay-line method is one of the techniques used to increase the effective beam length; a FabryPerot cavity is another one that is widely used. Although laser interferometric detectors have a much wider frequency band than resonant-mass antennae, the sensitivity at frequencies higher than $1 \mathrm{kHz}$ might be surpassed by that of a future resonant antenna [3].

LIGO-I is the biggest project in the world in the sense of its manpower and budget scale [4]. Two $4 \mathrm{~km}$ baseline interferometers are being built: one 


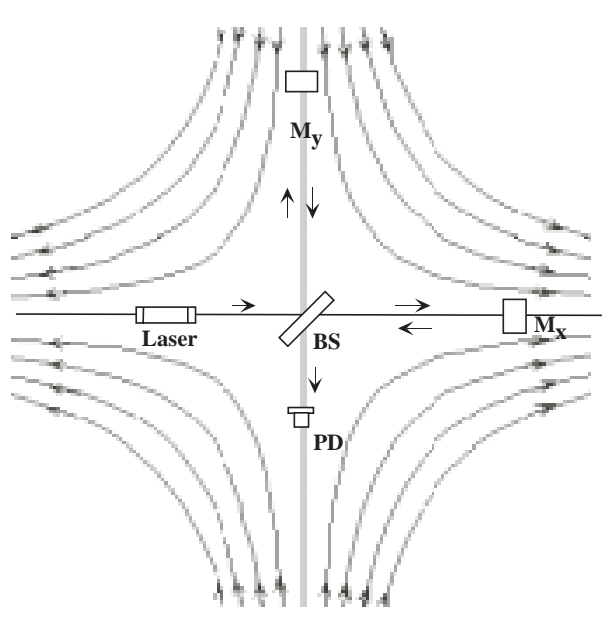

Figure 1. Laser interferometer sensing the passage of a gravitational wave by measuring the differential dispalcement of two orthogonal arms with high sensitivity.

in Hanford, Washington, and the other in Livingston, Louisiana. In September, 2001, all items in an in-vacuum installation were completed. At the Hanford site, a $2 \mathrm{~km}$ baseline interferometer has achieved locks in a recycled configuration for $1 \mathrm{hr}$ with a sensitivity of $10^{-18} \mathrm{rms}$. At the Livingston site, a $4 \mathrm{~km}$ baseline was locked as a Michelson interferometer with Fabry-Perot arms. At the Hanford site, commissioning started for a $4 \mathrm{~km}$ full-scale interferometer.

In Europe, a French and Italian project, VIRGO [5], has completed vacuum baking of its north arm in Pisa, Italy. The west arm, one of the three kilometer arms, is planned to be completed in June, 2002. The optical design of VIRGO is the same as that of LIGO-I and also that of the final state of TAMA. A test of the Michelson interferometer conducted in its center chamber achieved $5 \times 10^{-12} \mathrm{~m} / \sqrt{ } \mathrm{Hz}$ at $10 \mathrm{~Hz}$, which showed a high stability of a low-frequency anti-vibration system that characterizes the design of VIRGO. Also, a test of power recycling attained a gain of 100 using a $160 \mathrm{~mW}$ power laser source.

GEO is a collaboration between Germany and
England [6]. It does not adopt Fabry-Perot cavities for its optical configuration, but adopts both power and signal recycling applied to a basic Michelson interferometer with a $600 \mathrm{~m}$ baseline length. A $1 \mathrm{~W}$ power laser was injected to a series of mode cleaners, and an auto-alignment control circuit was tested using a one-arm system by the summer of 2001. A coincidence run with LIGO is planned for the end of 2001.

TAMA is a Japanese project with a $300 \mathrm{~m}$ baseline length built in Mitaka, Tokyo [7].

Among these projects, TAMA has attained week-long data taking with the highest sensitivity in the world. We would like to present the current activity of TAMA and research for LCGT, the $\mathrm{km}$ scale interferometer project in Japan.

\section{TAMA}

The objective of TAMA is to develop advanced technologies for a km-scale interferometer and to observe possible GW events in our Galaxy. The funding originally covered five years. Its construction started in April, 1995. A two-years extension was approved last year. Four years of research money has been scheduled for after this extension.

\subsection{Hardware of TAMA}

The optical configuration is shown in Fig. 2. Its has two Fabry-Perot cavities with a $300 \mathrm{~m}$ baseline length. Although the present configuration has no power recycling mirror, the installation is under going. A $10 \mathrm{~W} \mathrm{Nd}$ :YAG laser was introduced through a ring-type mode cleaner to the Michelson part of the interferometer. Optical phase modulation was applied upstream of the mode cleaner. Four main mirrors were suspended by a double suspension system on a 3-stage stack with sealed rubbers (Fig. 3).

The whole optics system, except for the laser source, was put inside an ultra-high-vacuum environment. Since the vacuum tubes were put in $2 \mathrm{~m}$ deep underground tunnels, only buildings for the center chamber and the two end chambers were seen on the ground of the site. The vacuum system had not been baked, but was processed by an electro-chemical-polishing technique [8]. 


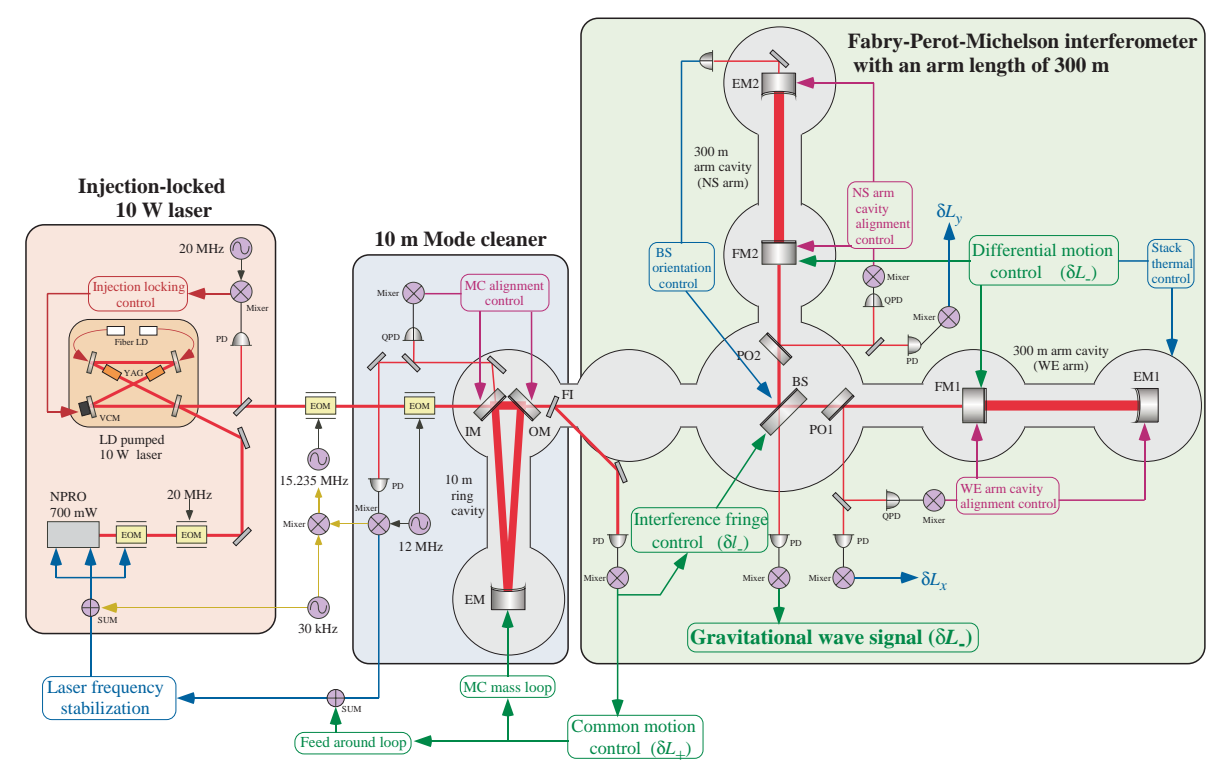

Figure 2. Optical configuration of TAMA.

\subsection{Operation of TAMA}

Since August, 1999, several week-long operations with data taking were performed as listed in Table 1 . In the earlier runs, the system characteristics were studied along with upgrading the feedback parameters for the optimum sensitivity. The data analysis of DT 2 was published [9]. DT 4 was the initial long observation in the original hardware, which was reported in [10].

Table 1

Operation with data taking

\begin{tabular}{lll}
\hline Run & Term & Hour \\
\hline DT 1 & 6-Aug $\rightarrow$ 7-Aug 1999 & 7 \\
DT 2 & 17-Sept $\rightarrow$ 20-Sept 1999 & 31 \\
DT 3 & 20-Apr $\rightarrow$ 23-Apr 2000 & 13 \\
DT 4 & 21-Aug $\rightarrow$ 4-Sept 2000 & 161 \\
DT 5 & 2-Mar $\rightarrow$ 8-Mar 2001 & 111 \\
DT 6 & 15-Aug $\rightarrow$ 20-Sept 2001 & 1038 \\
\hline
\end{tabular}

After DT 4, the anti-vibration system was upgraded by two hardware changes. One of them was adopting active pneumatic isolation systems, which were inserted between the legs of the main mirror vacuum chambers and the floor. The other was to decrease the eigenmode-frequencies of the test masses by adjusting the position of the suspension wires. An automatic recovery system of feedback loops had been gradually established until DT 6, which was the epoch-making observation by the first-phase run of TAMA.

Figure 4 shows the history of improving the sensitivity of the interferometer of TAMA. The dates given in the figure correspond to each operation in Table 1 (just before or after an operation). Except for the low-frequency region, the sensitivity is close to its target. A slight difference at the most sensitive frequency comes from an unidentified noise source.

The data taken in DT 6 are being analysed. A preliminary analysis of DT 6 showed that the scope of the TAMA range extends to $50 \mathrm{kpc}$ for BNS with a signal-to-noise ratio of 10 . 


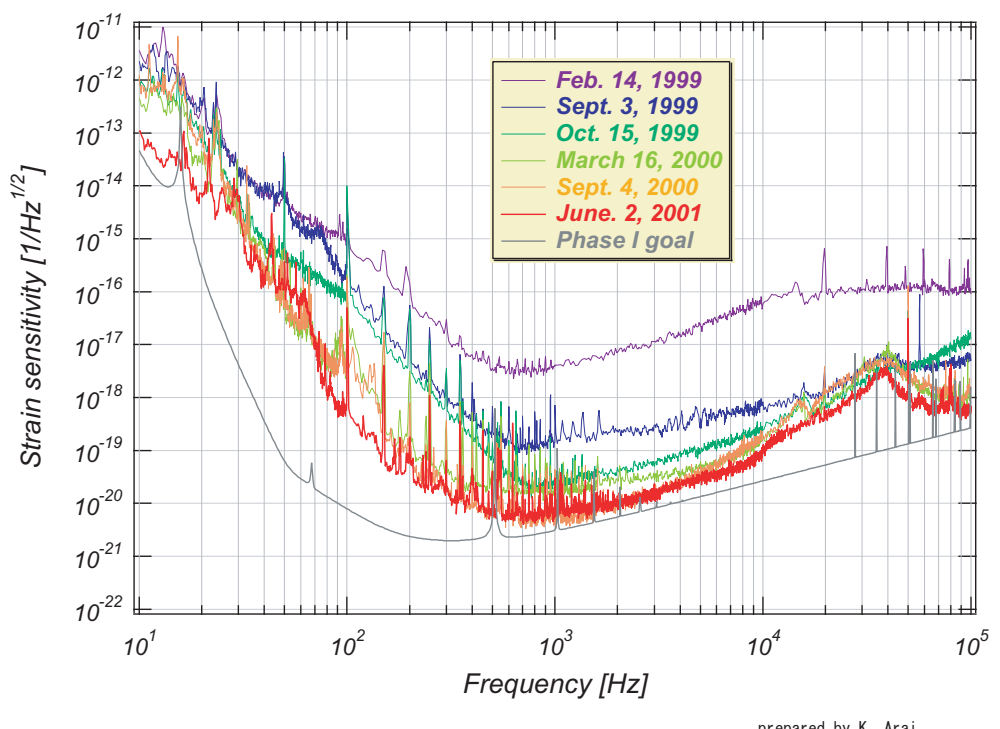

Figure 4. History of the sensitivity improvements of TAMA.

\section{LCGT}

Since BNS exists and the signal of the coalescence is precisely predicted, the event is the most important target of the ground-based interferometric detectors. However, since the event rate is $10^{-6}$ per year for such a mature galaxy as ours, we have to wait for 30 years on the average for the necessary sensitivity to reach the VIRGO cluster $(20 \mathrm{Mpc})$, because there is one galaxy per 1 cubic $\mathrm{Mpc}$ of space on the average. It is clear to everyone that it is necessary to develop more sensitive detectors to see more remote galaxies. By increasing the sensitivity by ten times, the above waiting time becomes 0.4 months. This is the reason why we need the Large-scale Cryogenic Gravitational wave Telescope (LCGT) [11], which is expected to be financed as soon as possible.

\subsection{Design of LCGT}

The original design of LCGT was made in 1996 and improved in 2001. The main design parameters are listed in Table 2.

The estimated sensitivity for the orthodox Fabry-Perot-Michelson interferometer with power
Table 2

LCGT design parameters

\begin{tabular}{ll}
\hline Item & Parameter \\
\hline Baseline length & $3 \mathrm{~km}$ \\
Laser Power & $100 \mathrm{~W}$ \\
Input power at BS & $5000 \mathrm{~W}$ \\
Finesse & 100 \\
Beam radius at & $3 \mathrm{~cm}$ \\
Main Mirror & Sapphire $51 \mathrm{~kg}, 30 \mathrm{~K}$ \\
Mechanical Q & $10^{8}$ \\
Suspension pendulum & $1 \mathrm{~Hz}, \mathrm{Q}=2 \times 10^{8}, 10 \mathrm{~K}$ \\
Anti-vibration & $10^{-3}$ at $1 \mathrm{~Hz}$ \\
\hline
\end{tabular}

recycling is shown in Fig. 5 along with other projects, including TAMA. The sensitivity of the advanced LIGO [12] is better than that of LCGT in a frequency range limited by the photon shot noise, which is realized by resonant sideband extraction. LCGT is constructed in the mine of Kamioka near to Super-KAMIOKANDE.

If LCGT can attain its planned sensitivity, the signal of the coalescence of BNS at $200 \mathrm{Mpc}$ can be detected several times every year. If we focus 


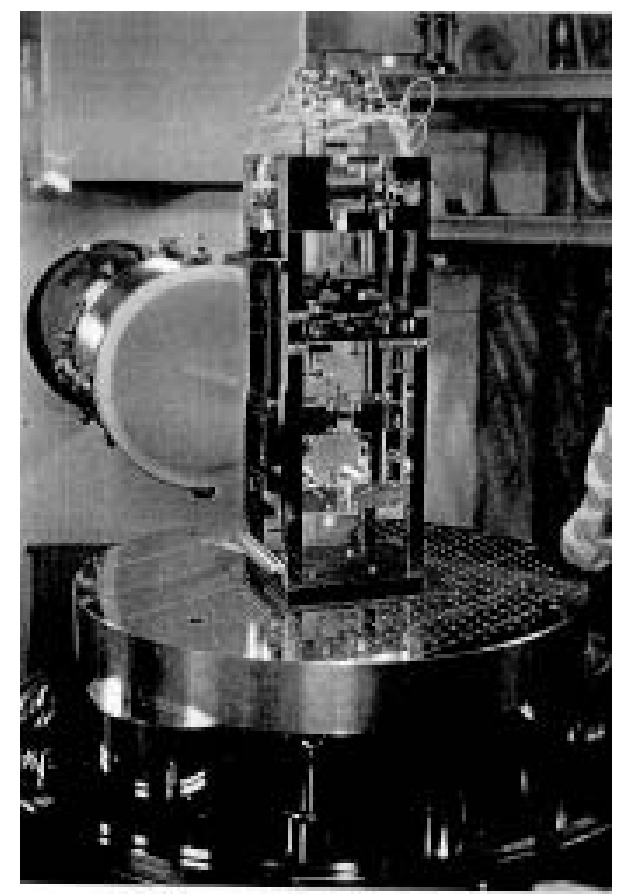

Figure 3. Mirror suspension sytem on a stack system. The main mirrors are suspended by a double suspension system with magnetic damping of the second mass.

our attention on the coalescence of $\mathrm{BBH}$, the $\mathrm{S} / \mathrm{N}$ ratio of the signal would become larger, and an event 1 Gpc away could be easily detected.

\subsection{R\&D for LCGT}

The special feature of LCGT is the adoption of a cryogenic mirror to try to reduce thermal noise around $100 \mathrm{~Hz}$. Another feature is the adoption of an underground site. First, the underground site was investigated using a $20 \mathrm{~m}$ locked FabryPerot interferometer for stable operation and its environmental characteristics. Surprisingly, the seismic noise in the mine is less by two orders than that at the TAMA site around the frequency of the suspension pendulum eigenmode-frequency $(\sim 1 \mathrm{~Hz})$. We have obtained proof of the high stability of the underground site [13]. The stability comes both from the quieter seismic noise of the

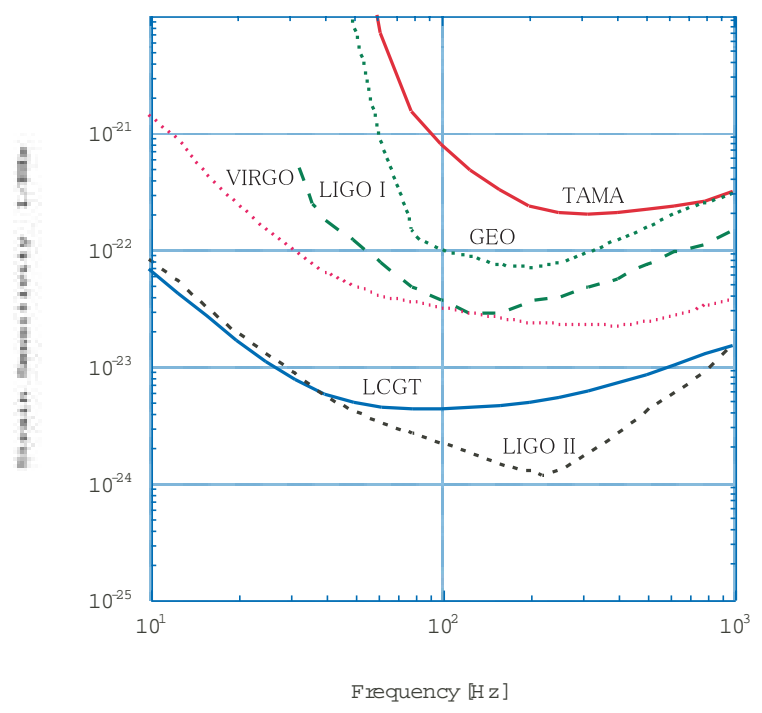

Figure 5. Design sensitivity of LCGT along with other projects.

surrounding hard rock and from the high stability of the temperature during the year ( $15 \pm 1$ degree).

Figure 6 shows a schematic of a cryogenic mirror used as the end mirror of the Fabry-Perot cavity. The mirror is suspended and thermally shielded. Since the vacuum tube for the laser beam is maintained at room temperature, a long radiation shield is needed to prevent hot gas molecules from coming into the mirror. A lowfrequency anti-vibration system is mounted outside of the cryogenic temperature part, but still inside the vacuum.

One of essential problems concerning the cryogenic mirror was how to cool it under a condition of high heat production due to laser light loss in the mirror. The production rate depends on both the qualtiy of the light-reflective coating and the internal optical loss of the mirror substrate. The design of LCGT adopts a sapphire test mass (mirror substrate) suspended by two sapphire fibers. Since sapphire has a high heat conductivity at the cryogenic temperature, the suspension fiber can effectively extract heat from the mirror. This 


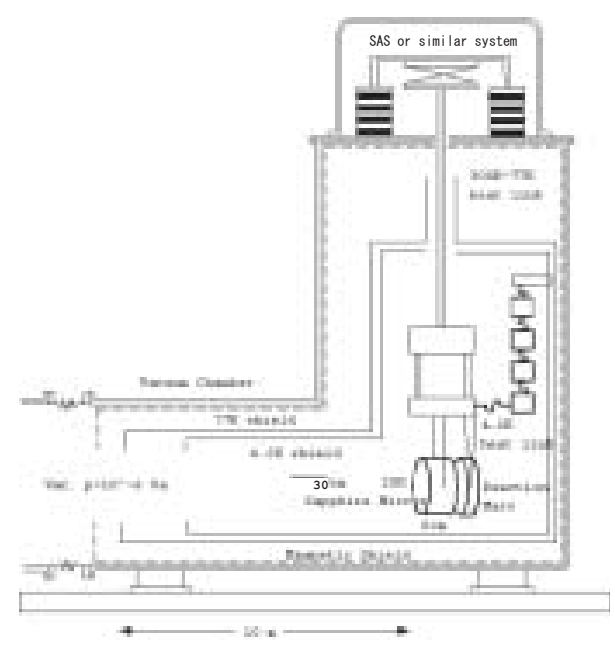

Figure 6. Schematic diagram of the cryogenic mirror for LCGT.

was proved by an experiment under some conditions [14]. Since LCGT adopts Fabry-Perot cavities, a light beam passes inside the front mirror while light does not pass through the end mirror. Therefore, heat production is higher in the front mirror. In the design of LCGT, the power passing through the substrate is $2.5 \mathrm{~kW}$ and the stored light power in the cavity is $160 \mathrm{~kW}$. The optical loss in the reflective coating can be less than $0.1 \mathrm{ppm}$, which will produce $16 \mathrm{~mW}$. Contrary to this controllable heat, there is a much larger heat production due to the optical loss of the sapphire, which has been reported to be at least $40 \mathrm{ppm} / \mathrm{cm}$. LCGT needs $18 \mathrm{~cm}$ thickness of the mirror substrate in order to maintain its mechanical resonances higher against thermal noise, which produces at least $1.8 \mathrm{~W}$. Since the heat power of a commercially available refrigerator is $700 \mathrm{~mW}$ at $5 \mathrm{~K}$, this causes some difficulty. We expect that this difficulty can be conquered by reducing the optical loss of the sapphire or reducing the light power passing through inside of the mirror substrate by applying another optical configuration of the interferometer, such as resonantsideband-extraction. Although we seek its clear solution, we can expect that it would not become fatal, but can be solved in a design process.

The second problem of the cryogenic mirror was mechanical loss arising from an additive mechanism for cooling or intrinsic loss in the cryogenic temperature. We measured both the internal loss of the mirror substrate and the pendulum loss of the suspension fiber and proved that all of these losses decreased with the cryogenic temperature, which means that the thermal noise is reduced in two ways: a decrease in the temperature and a decrease in the mechanical losses. [15]

The third problem of this design was that the low-temperature mirror attracted residual gas molecules onto its surface, which would reduce the optical quality of the mirror. We examined it using a high-finesse Fabry-Perot cavity placed inside a vacuum cryostat, and measured the change in the finesse (that is the function of the optical reflectivity) for two months. We could observe a slight decrease in the finesse, and concluded that the change can be safely coped with by an appropriate operational procedure. Needless to say, one of the mirrors forming the Fabry-Perot cavity faced the hot inner surface of the vacuum chamber to simulate the real configuration.[16]

The above three results of $R \& D$ for a cryogenic mirror confirmed that we can in principle make a cryogenic laser interferometer. During the course of this study, we found that cryogenics can solve the problem of the thermal-lens effect inside the mirror, which is the main obstacle in future interferometers working at room temperature.[17]

The next step of R\&D for LCGT is to establish a cryogenic interferometer, itself. For this objective, a suspension prototype, including the method of cooling, was tested at the Kashiwa campus of ICRR, the University of Tokyo (shown in Fig 7). We have not encountered any serious problem so far concerning this practical system.

The above research is a part of the $R \& D$ for LCGT. There are many acitivities that are not treated here. They include a basic test of a resonant-sideband-extraction technique, the development of a pure crystal-growth technique, a test of a new alloy material with high mechanical damping, a calorimetric measurement of the sapphire optical loss, collaborative research work 


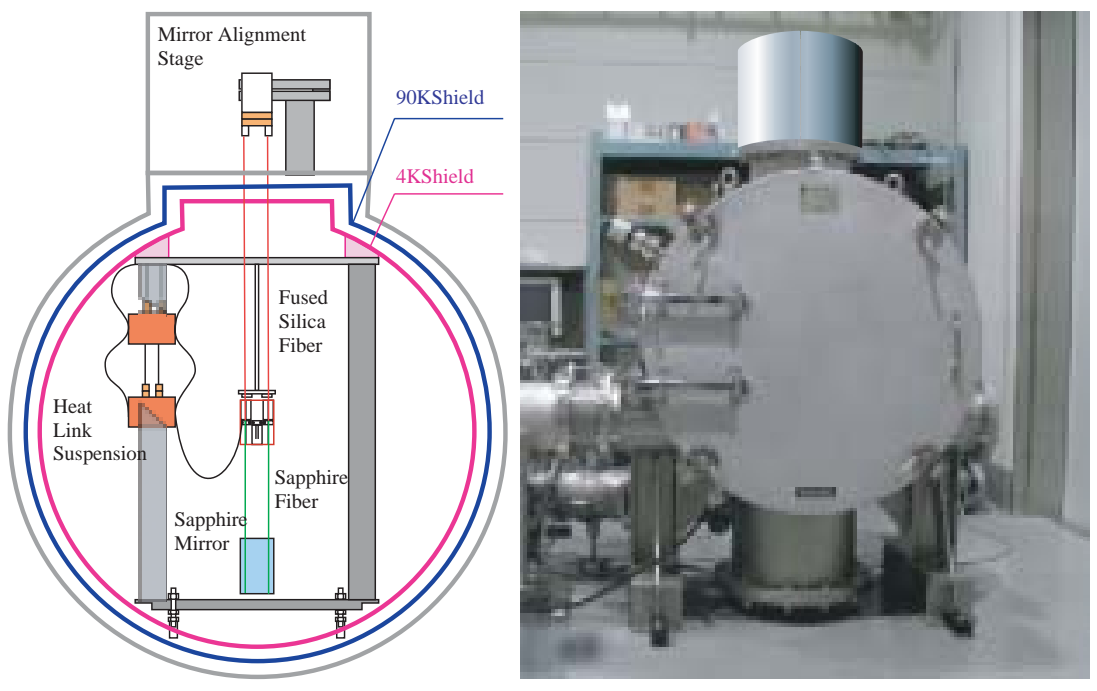

Figure 7. Practical test of a suspension system for a cryogenic interferometer at the Kashwia campus of ICRR.

on SAS with a group at Calfornia Institute of Technology, and collaborative research on a new devise for cryogenic suspension and a high-power laser with groups of the Australian Consortium for Interferometric Gravitational Astronomy.

\section{Conclusion}

More than $1000 \mathrm{hr}$ of continuous data have been accumulated in data taking of an intermediate-scale laser interferometric gravitational wave detector, TAMA. Also, R\&D for a cryogenic mirror has been promising. Both of these results make us confident to begin a fullscale interferometer project, LCGT, in Japan. Interferometer observations over world-wide scale for GW will start in a few years and will produce productive results soon.

\section{REFERENCES}

1. J.H.Taylor and J.M.Weisberg, Astrophys. J. 345 (1989) 434.

2. G.A.Prodi, in 4th Edoardo Amaldi Conference on GW, July 2001, Perth.
3. M.Cerdonio, et al., Phys. Rev. Lett. 87 (2001) 031101-1.

4. A.Abramovici, et al., Phys. Lett. A218 (1996) 157.

5. The VIRGO Collaboration, VIRGO Final Conceptual Design (1992).

6. K.Danzmann, et al., Max-Plank-Institut fur Quantenoptik Report, p190 (1994).

7. K.Tsubono, in Proceedings of the 1st Amaldi Conference on Gravitational wave Experiments, p112 (1995).

8. Y. Saito, et al., Vacuum 53 (1999) 353.

9. H.Tagoshi, et al., Phys. Rev. D63 (2001)062001.

10. M.Ando, et al., Phys. Rev. Lett. 86 (2001) 3950.

11. K.Kuroda, et al., Int. J. Mod. Phys. D8 (1999) 557.

12. http://www.ligo.caltech.edu/LIGO_web/lsc/lsc.html

13. in preparing for publication.

14. T.Uchiyama, et al., Phys. Lett. A242 (1998) 211.

15. T.Uchiyama, et al., Phys. Lett. A273 (2000) 310.

16. S.Miyoki, et al., Cryogenics 41 (2001) 415.

17. T.Tomaru, Doctor Thesis, The University of Tokyo (2001). 